\title{
Benchmarking of FLUKA production cross sections of positron emission to- mography isotopes for in-vivo range verification in hadron therapy
}

\author{
Giulia Aricò ${ }^{1, *}$, Giuseppe Battistoni ${ }^{2,3}$, Francesco Cerutti ${ }^{1}$, Felix Horst $^{4,5}$, Andrea Mairani ${ }^{6,7}$, Christoph Schuy $^{4}$, Uli \\ Weber $^{4}$, and Alfredo Ferrari ${ }^{1}$ \\ ${ }^{1}$ European Organization for Nuclear Research (CERN), Geneva, Switzerland \\ ${ }^{2}$ National Institute for Nuclear Physics (INFN), Milan, Italy \\ ${ }^{3}$ Trento Institute for Fundamental Physics and Application (TIFPA), Trento, Italy \\ ${ }^{4}$ GSI Helmholtz Centre for Heavy Ion Research, Darmstadt (GSI), Germany \\ ${ }^{5}$ Technische Hochschule Mittelhessen (THM), Giessen, Germany \\ ${ }^{6}$ Heidelberg Ion-Beam Therapy center (HIT), Heidelberg, Germany \\ ${ }^{7}$ National Centre of Oncological Hadrontherapy (CNAO), Pavia, Italy
}

\begin{abstract}
Protons and carbon ions have been extensively used for radiotherapy treatments, and in comparison to conventional radiotherapy, they allow a more conformal dose to the target tumor, especially in case of deep-seated tumors. However, the accuracy of hadron therapy treatments is affected by uncertainties in the particle range calculations. Several techniques are under development for in-vivo range verification, one of which consists on measuring the activity distributions of positron emitters, such as ${ }^{10} \mathrm{C},{ }^{11} \mathrm{C}$ and ${ }^{15} \mathrm{O}$, which are produced in the patient body during proton and carbon ion treatments. A comparison between measured and expected positron emitter activity distributions can provide information on the quality of the delivered treatment and accuracy of the particle range calculations. In this work the FLUKA production cross sections for ${ }^{10} \mathrm{C},{ }^{11} \mathrm{C}$ and ${ }^{15} \mathrm{O}$ originated from proton and carbon ion beams in carbon and oxygen targets were compared with experimental data, at low and therapeutic energies.
\end{abstract}

\section{Introduction}

Unlike photons, the depth-dose profile of light ions is characterized by an initial low plateau and a so called Bragg peak at the end of the particle range. As a result, especially in the case of deep-seated tumors, hadron therapy can offer an improved dose conformation to the tumor and a better sparing of the surrounding healthy tissues in comparison to conventional radiotherapy. Furthermore, heavier ions possess a higher biological effectiveness; this makes carbon ions particularly suitable for treating radio-resistant tumors [1].

To assure a full coverage of the target with the prescribed dose, safety margins are considered around the tumor, to take into account, among other factors, uncertainties on the patient positioning and particle range calculations [2]. Particle ranges are calculated based on the information provided by the patient $\mathrm{x}$-ray CT image acquired for treatment planning for each patient. However, uncertainties in the calibration of the CT scan, physics models, patient positioning, organ motion and heterogeneities (e.g. in the nasal cavity or in the bladder) reduce the accuracy of the particle range estimation. Moreover, secondary fragments are produced as a consequence of non-elastic reactions in the patient tissues' nuclei. These fragments represent in general an issue, as they can be particularly damaging for the

*e-mail: giulia.arico@cern.ch healthy tissues surrounding the tumor [3]. However, they can also be exploited for in-vivo range verification. For instance, ${ }^{10} \mathrm{C},{ }^{11} \mathrm{C}$ and ${ }^{15} \mathrm{O}$ are produced along the path of the primary protons and carbon ions; being $\beta^{+}$emitters, their activity profile can be measured by positron emission tomography (PET). PET measures the anti-parallel $511 \mathrm{KeV}$ photons that are produced as a result of the annihilation of a positron, emitted in the $\beta^{+}$decay, and an electron, present in the patient body.

In the case of proton beams, range estimation is more challenging; as shown in [4], the 50\% distal level of the activity profile measured by PET may differ up to $6 \mathrm{~mm}$ by the proton range. This is due to the absence of projectile fragments contributing to the distal part of the activity distribution. Moreover, target fragments are produced only above the energy threshold of the fragmentation reaction. As a result, the end of the activity profile is shifted by few $\mathrm{mm}$ with respect to the position of the Bragg peak. Instead, for carbon ion beams, ${ }^{10} \mathrm{C}$ and ${ }^{11} \mathrm{C}$ are produced both from the target and the projectile, and the correlation between the end of the activity distribution and the primary particle range is much closer [5].

A comparison between positron emitter activity distributions measured by PET and predicted by Monte Carlo simulations provide information on the accuracy of the particle range and dose calculations [6]. This procedure allows real time treatment monitoring, or verification of the deliv- 
ered dose with respect to the planned dose after the treatment delivery. Indeed, ${ }^{10} \mathrm{C}$ and ${ }^{15} \mathrm{O}$ have rather short half lives (19.29 s and $122.24 \mathrm{~s}$, respectively) and can therefore be measured in-beam (online), whereas ${ }^{11} \mathrm{C}$ has a longer half life (20.334 $\mathrm{min}$ ) and it is mainly used for measurements after the treatment delivery (offline).

Range monitoring techniques rely on accurate modeling of nuclear interaction and ion fragmentation in the used Monte Carlo code. In the present work, the FLUKA production cross sections of PET isotopes $\left({ }^{10} \mathrm{C},{ }^{11} \mathrm{C}\right.$, and ${ }^{15} \mathrm{O}$ ) were benchmarked against experimental data recently measured at the Marburg Ion-Beam Therapy center [7] and against available literature data.

\section{Material and Method}

\subsection{The FLUKA code}

FLUKA $[8,9]$ is a general purpose Monte Carlo code developed by a CERN-INFN collaboration, for simulations of particle transport and interactions with matter. FLUKA follows the so called "microscopic" approach whenever possible, i.e. particle production data at a single interaction level are used as reference. Nucleus-nucleus collisions at energies below $125 \pm 25 \mathrm{MeV} / \mathrm{u}$ are handled in FLUKA by a model based on the Boltzmann master equation (BME) [10]. Depending on the impact parameters, collisions are medeled as complete fusion (low impact parameter), three body mechanism (more peripheral collisions), and single nucleon mode break-up/transfer (high impact parameter) [11]. For nucleus-nucleus collisions at energies between $125 \pm 25 \mathrm{MeV} / \mathrm{u}$ and $5 \mathrm{GeV} / \mathrm{u}$ an extensively modified and improved version [12] of the relativistic quantum molecular dynamic rQMD-2.4 (original code from [13]) has to be linked in FLUKA for accurate modeling of the dynamic part of the nucleus-nucleus collisions. At the end of the reaction, the excited nucleus can dissipate the residual energy by means of evaporation, fission, de-excitation and Fermi break-up processes. In case of proton (hadron) induced reactions, the production of secondary composite fragments, like deuteron, tritium, helium-3 and alpha particles, is described in FLUKA first by coalescence, followed by evaporation or Fermi breakup equilibrium stages, which are common to the nucleusnucleus case [11]. Coalescence is activated by means of a PHYSICS card.

FLUKA is used in hadron therapy to generate the basic nuclear reaction database, and to validate the dose calculations as a support to the analytical treatment planning systems [11]. Furthermore, FLUKA is used at CNAO as reference for the PET activity measurements [14].

\subsection{Cross section studies in FLUKA}

The FLUKA production cross sections of the PET isotopes ${ }^{10} \mathrm{C},{ }^{11} \mathrm{C}$ and ${ }^{15} \mathrm{O}$ originated from protons and carbon ions in carbon and oxygen targets at energies of interest for hadron therapy, were benchmarked against experimental data. The natural composition of ${ }^{12} \mathrm{C}$ and ${ }^{16} \mathrm{O}$ was considered in FLUKA to model the targets. Only target-like fragments were considered, for a direct comparison with the experimental data. The most recent FLUKA development version (2018.1) was used.

\section{Results and Discussion}

\subsection{Proton beams}

Figure 1 shows the FLUKA production cross sections of ${ }^{10} \mathrm{C},{ }^{11} \mathrm{C}$ and ${ }^{15} \mathrm{O}$ produced by protons in carbon and oxygen, as a function of energy, compared to experimental data. Some differences were found for the short-lived isotopes. In particular, FLUKA underestimates (up to 25\%) the production cross sections of ${ }^{10} \mathrm{C}$ for beam energies below $100 \mathrm{MeV}$ (figure 1(a)), and overestimates (up to 27\%) the production cross sections of ${ }^{15} \mathrm{O}$ above $140 \mathrm{MeV}$ (figure $1(\mathrm{c})$ ). For ${ }^{11} \mathrm{C}$ (figure $1(\mathrm{~b})$ ), the agreement between FLUKA and experiments is satisfactory for all energies of interest for hadron therapy. Improvements in the FLUKA physics models will be carried out, in order to reduce the differences observed between experiments and simulations in the case of ${ }^{10} \mathrm{C}$ and ${ }^{15} \mathrm{O}$.

This work complements the results shown in [11], where production cross sections for the channels ${ }^{12} \mathrm{C}(\mathrm{p}, \mathrm{pn})^{11} \mathrm{C}$, and ${ }^{16} \mathrm{O}(\mathrm{p}, \mathrm{pn}){ }^{15} \mathrm{O}$ were shown with a previous FLUKA development version and with less experimental data. Valuable comparisons between FLUKA predictions and $\beta^{+}$activity depth profiles produced by protons in PMMA targets at therapeutic energies were carried out in $[4,15]$; FLUKA was shown to be a valid tool for calculation of the activity distributions for offline PET measurements for proton therapy.

\subsection{Carbon ion beams}

The production cross sections of PET isotopes produced by carbon ion beams have not been as extensively investigated as for protons. Figure 2 shows the FLUKA production cross sections of ${ }^{10} \mathrm{C}$ and ${ }^{11} \mathrm{C}$ produced by carbon ions in carbon as a function of energy, compared to the available experimental data. The experimental data are in rather good agreement among them, except for the data points in [16], which are significantly higher with respect to [7] at similar energies. Regarding FLUKA, the production cross sections of ${ }^{10} \mathrm{C}$ are in good agreement with the data in [7] for beam energies below $100 \mathrm{MeV} / \mathrm{u}$, whereas differences of about $50 \%$ can be observed for energies above $100 \mathrm{MeV} / \mathrm{u}$. For ${ }^{11} \mathrm{C}$, a satisfactory agreement between FLUKA and the data in $[7,17]$ was found for energies below $160 \mathrm{MeV} / \mathrm{u}$, whereas for higher energies FLUKA underestimates the production cross sections by about $25 \%$. The observed differences do not have a critical impact on the delivered dose calculations, for which FLUKA has been demonstrated to satisfy the clinical requirements [11]. Moreover, good agreement between measured and simulated $\beta^{+}$activity distributions produced by 260 $\mathrm{MeV} / \mathrm{u}$ carbon ion beams in PMMA targets was found in [18]. Nevertheless, based on the findings shown here, refinements of the FLUKA physics models will be carried out, and their effects on PET monitoring technique will be investigated. 

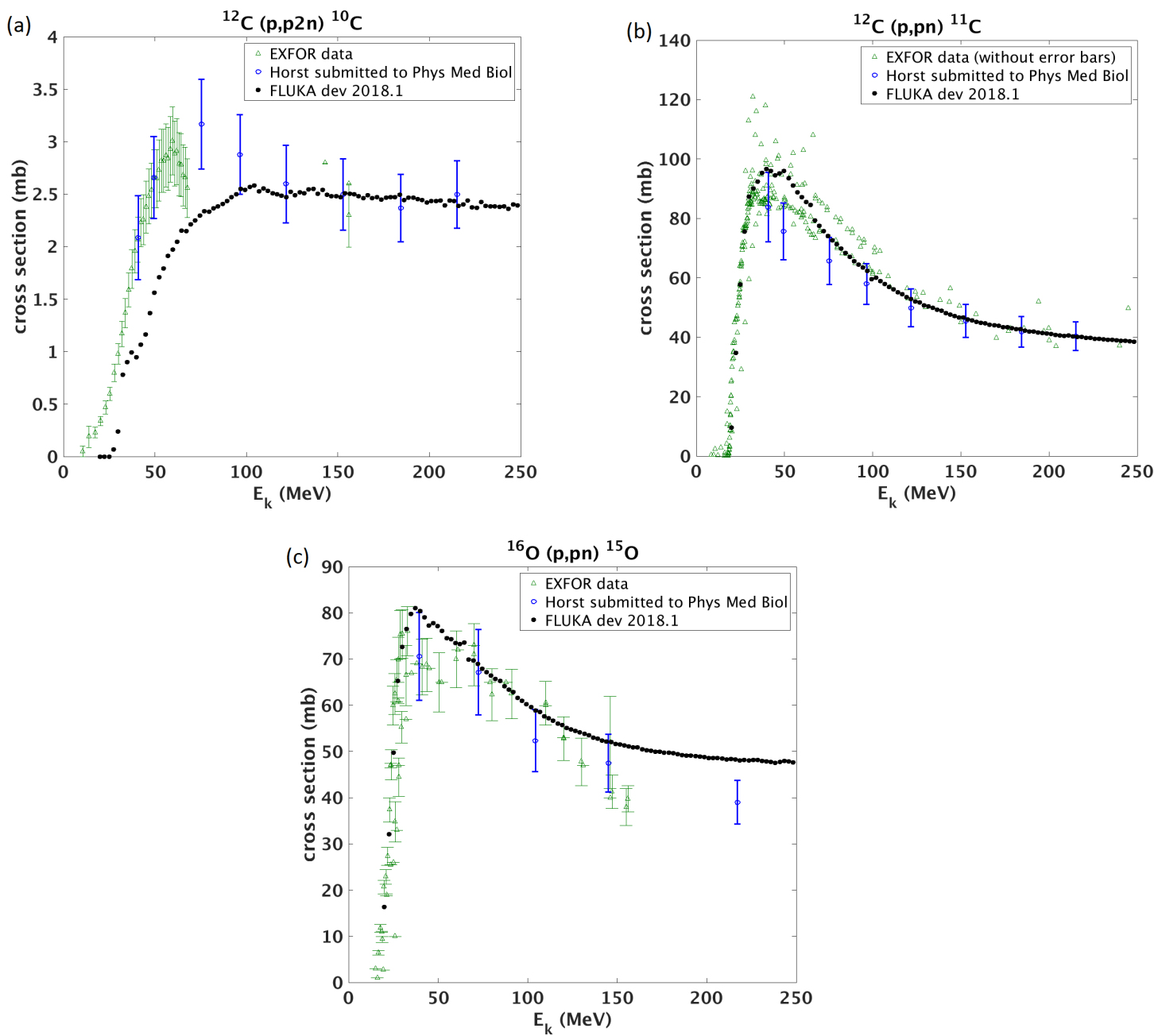

Figure 1. Production cross sections for ${ }^{10} \mathrm{C},{ }^{11} \mathrm{C}$ and ${ }^{15} \mathrm{O}$ originated from proton beams in carbon and oxygen targets as a function of energy. The black filled dots are FLUKA simulations, the green empty symbols are data from the Experimental Nuclear Reaction Data (EXFOR) library, and the blue empty circles are data from [7]. For the reaction ${ }^{12} \mathrm{C}(\mathrm{p}, \mathrm{pn}){ }^{11} \mathrm{C}$ the experimental error bars are not shown for the sake of image clearness.

(a)

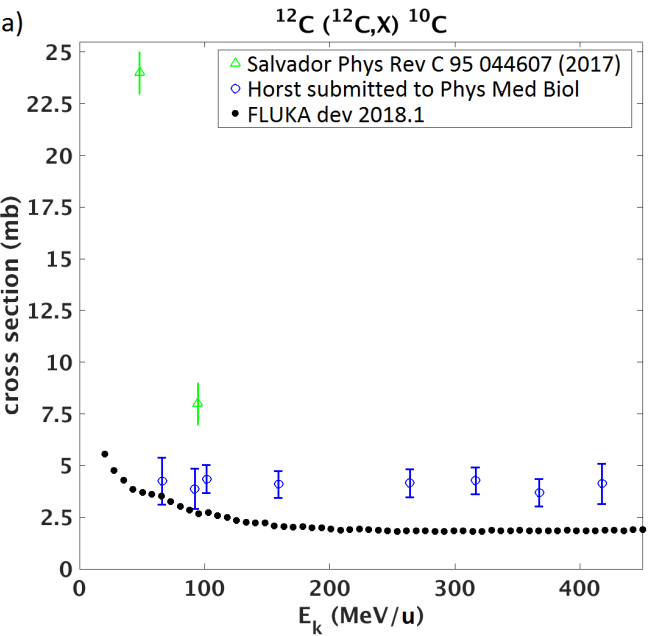

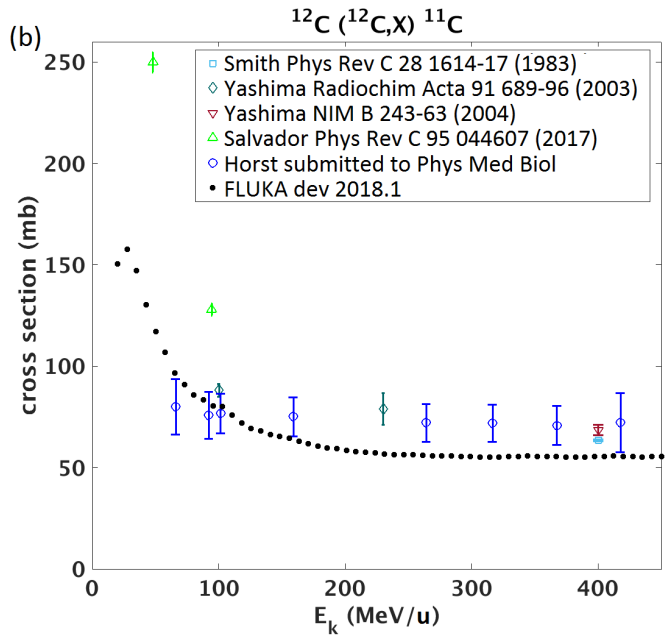

Figure 2. Production cross sections for ${ }^{10} \mathrm{C}$ and ${ }^{11} \mathrm{C}$ originated from carbon ion beams in carbon targets as a function of energy. The black filled dots are FLUKA simulations, the empty symbols are experimental data [7, 16, 17, 19, 20]. 


\section{Conclusions}

Benchmarking of FLUKA against cross section data is crucial for the development of the physics models embedded in the code. General results and predictions are naturally derived by the physics models, with minimal free parameters. In this work, the FLUKA cross sections for the reactions ${ }^{12} \mathrm{C}(\mathrm{p}, \mathrm{p} 2 \mathrm{n}){ }^{10} \mathrm{C},{ }^{12} \mathrm{C}(\mathrm{p}, \mathrm{pn}){ }^{11} \mathrm{C},{ }^{16} \mathrm{O}(\mathrm{p}, \mathrm{pn}){ }^{15} \mathrm{O}$, ${ }^{12} \mathrm{C}\left({ }^{12} \mathrm{C}, \mathrm{X}\right){ }^{10} \mathrm{C}$ and ${ }^{12} \mathrm{C}\left({ }^{12} \mathrm{C}, \mathrm{X}\right){ }^{11} \mathrm{C}$ were compared with experimental data. Such reactions are important as the activity distributions of ${ }^{10} \mathrm{C},{ }^{11} \mathrm{C}$ and ${ }^{15} \mathrm{O}$, measured by PET scanners either during or shortly after the radiotherapy treatment, can be used for in-vivo range verification. FLUKA has been shown to predict satisfactorily well the activity distributions of PET isotopes, both for protons and carbon ions in PMMA targets [4, 15, 18]. The present level of agreement is sufficient for semi-quantitative predictions, and it can already be suitable for comparing the shape of predicted and measured activity distributions. This work highlights that some improvements in the FLUKA physics models can be performed in order to achieve an even better agreement with the experimental production cross sections. The effects of such improvements on the activation yields and profile distributions should finally be assessed in clinical scenarios. This research will contribute to improve the radiotherapy treatments at CNAO, where FLUKA is used as reference for the PET measurements.

\section{Acknowledgments}

GA wishes to thank the European Union's Horizon 2020 research and innovation programme under the Marie Sklodowska-Curie grant agreement No 675265 OMA Optimisation of Medical Accelerators - for funding her research project.

\section{References}

[1] Jäkel O, Radiat Prot Dosimetry 137 156-166 (2009)

[2] Paganetti H, Phys. Med. Biol. 57(11) R99-R117 (2012)

[3] Kraan AC, Front. Oncol. 5:150 (2015)

[4] Parodi K et al., Phys. Med. Biol. 47 21-36 (2002)

[5] Weber and Kraft, The Cancer Journal 15(4) (2009)

[6] Parodi K and Polf JC, Med. Phys. 45(11) e1036-50 (2018)

[7] Horst F, Adi W, Aricò G et al., Phys. Med. Biol. 64 205012 (2019)

[8] Ferrari A et al., CERN-2005-10, INFN/TC-05/11, SLAC-R-773 (2005)

[9] Böhlen TT et al., Nuclear Data Sheets 120 211-14 (2014)

[10] Cerutti F et al., Proceeding 11th Int. Conf. on Nucl. React. Mech. Varenna, Italy, 12-16 June, pp 507-11 (2006)

[11] Battistoni G et al., Frontiers in Oncology 6116 (2016)

[12] Andersen V et al., Advances in Space Research, 34(6) 1302-1310 (2004)

[13] Sorge H, Phys. Rev. C 52 3291-3314 (1995)

[14] Fiorina E, Nuclear Instruments and Methods in Physics Research A 824 198-201 (2016)

[15] Rosso V et al., JINST 8 C03021 (2013)

[16] Salvador S et al., Phys. Rev. C 95044607 (2017)

[17] Yashima H et al., Radiochim. Acta 91 689-96 (2003)

[18] Sommerer F, PhD Thesis, University of Vienna (2007)

[19] Smith AR et al., Phys. Rev. C 95044607 (1983)

[20] Yashima H et al., Nucl. Instr. and Meth. in Phys. Res. B 226 243-63 (2004) 